Pak. j. sci. ind. res. Ser. B: biol. sci. 2018 61B(1) 47-51

\title{
Comparative Study of Tribolium castaneum H. (Coleoptera: Tenebrionidae) Occurred on Different Wheat Varieties in Pakistan
}

\author{
Muhammad Waqar Hassan*, Javaid Iqbal and Moazzam Jamil \\ University College of Agriculture and Environmental Sciences, \\ The Islamia University of Bahawalpur, Pakistan
}

(received October 18, 2014; revised June 12, 2017; accepted July 11, 2017)

\begin{abstract}
Five wheat varieties i.e., AASS, CHAKWAL, FARID, MIRAJ and GALAXY in Pakistan were campared to survey the red flour beetle population occurrence during one month period. Only sound grains were taken as medium of population growth for the red flour beetles. Study parameters included number of grains per unit weight, beetles population growth/survival percentage in relation to different wheat varieties and percent weight loss, if any in grains of these wheat varieties due to beetles feeding. Wheat varieties had significantly different number of grains per unit weight. Red flour beetles' population growth was checked in a 30 day time period. Beetles could not grow within the food medium of sound wheat grains of these varieties in this time span and most of these died. Survival percentage of beetles was measured in relation to different wheat varieties which did not vary significantly for these varieties. Within a given span of time percent weight loss due to beetle feeding in grains was nominal in all varieties and it also did not vary significantly between varieties. From these results it is concluded that red flour beetles being secondary stored grain insect pests were unable to multiply on sound grain wheat varieties and caused minimal weight loss in these varieties.
\end{abstract}

Keywords: wheat varieties, number of grains, weight loss, storage insects, red flour beetle, population growth

\section{Introduction}

Annual post-harvest losses of stored cereals range from $10-20 \%$ of the overall production, in which insect damage, microbial deterioration and other factors play the major role (Phillips and Throne, 2010). A primary factor in these losses is due to ravages of stored product insect pests that can reduce the quality and quantity of grains (Weaver and Subramanyam, 2000). Stored product insects have been associated with human activities since the earliest civilizations, and methods for their diagnosis and control have been reported for over a century (Levinson and Levinson, 1985). There are many safe, effective, and relatively simple prevention and control methods available to manage populations of storedproduct insect pests without the use of chemical insecticides (Phillips and Throne, 2010). In integrated pest management system, alongside other management practices, host plant resistance against different insect pests is very important part to effectively reduce pest populations without the indiscriminate use of toxic insecticides. It has been reported that varietal resistance is one of the basic components that should be seriously taken into account when a stored-product integrated pest management-based strategy is planned (Throne et al., 2000). It has been stated that substrate type,

*Author for correspondence; E-mail: waqar_722@hotmail.com germplasm and cracked kernels in the stored materials should be give due care for the management of serious stored grain insect pests (Athanassiou et al., 2010).

Wheat is staple food for the people in many countries across the globe. In Pakistan wheat is harvested in May and is stored for the entire season during which it is likely to be infested by different stored grain insect pests. Presence of broken grains in stored wheat is either an indication of an earlier infestation by primary pests (Arbogast et al., 2000) or otherwise it may be due to physical or mechanical injuries met with during harvesting, transport or storage process. As soon as a cereal contains cracked grains it may become vulnerable to attack by secondary stored product pest insects which primarily feed on broken grains.

Red flour beetle Tribolium castaneum Herbst (Coleoptera: Tenebrionidae) is a cosmopolitan pest of wheat especially wheat by-products. Its presence in wheat flour makes it responsible for change in colour and odour and is also pollute the host with its excreta and cast skins (Bosly and Kawanna, 2014). Consumption of the contaminated edibles can cause serious health issues (Gorham, 1979; 1975). Red flour beetles are primarily secondary stored product pests which can rarely feed or damage sound grains of cereals (Walter, 1990). However other authors indicated that red flour 
beetles over a certain period of time caused noticeable damage and weight loss to sound wheat grains in different wheat varieties and its population growth occurred in sound grains of wheat over a given time span (Ali et al., 2012; 2009).

This study was therefore started to check red flour beetles population increase or decrease in a 30 day time period and any weight loss in five different wheat varieties namely i.e., AASS, CHAKWAL, FARID, GALAXY and MIRAJ containing sound grains. Results would reveal the ability of red flour beetles to make damage and weight loss to different wheat varieties having sound grains during this one month period keeping in view the sound nature of grains in this study. Unlike some primary storage pest insects whose feeding makes substantial amount of damage in sound grains, the red flour beetle may cause minimal damage during this short period of one month. Therefore, even a small weight loss after one month period may indicate feeding on sound grains and damage to them by red flour beetles. Results would reveal relative feeding and damage as weight loss by red flour beetles in five wheat varieties over one month period. Any significant difference in weight loss among these varieties shall reveal some resistant or susceptible germplasm (s) or varieties relative to each other against this pest that may pinpoint their importance for their inclusion in integrated control programme for this pest in wheat showing resistance at this stage

\section{Materials and Methods}

Insect source. Red flour beetles were taken from laboratory culture maintained at $30 \pm 2{ }^{\circ} \mathrm{C}$ and $65 \pm 5 \%$ R.H., in Department of Entomology, University College of Agriculture and Environmental Sciences, The Islamia University of Bahawalpur. Adults of T. castaneum were taken and allowed to lay eggs in separate plastic jars containing sterilized whole wheat flour for a period of two weeks. After that adults were sifted out and flour containing eggs of T. castaneum was left for egg development inside the laboratory. These jars were kept for about one month for adult emergence. After one month from the date of sifting parent adults, emerging adults were of homogenous age and about one week or so older because according to Rees (2007) red flour beetles take about 25 days from egg to adult at $32.5^{\circ} \mathrm{C}$ and $>70$ relative humidity.

Experimental-setup: Five wheat varieties namely i.e., AASS, CHAKWAL, FARID, GALAXY and MIRAJ were obtained from Regional Agriculture Research Institute, (RARI) Bahawalpur, Pakistan. Experiments were performed to execute the current research aim. In first experiment $5 \mathrm{~g}$ weight measured with sensitive electrical weighing balance was taken for all varieties. Number of grains per $5 \mathrm{~g}$ weight was counted for every variety. This process was replicated four times to get mean values. In second experiment 10 adult red flour beetles (We could use 10 same age adult beetles sample size due to scarcity of culture) in the same day-age were released into $5 \mathrm{~g}$ weight of each variety (only sound grains per $5 \mathrm{~g}$ were used in experiments). This experimental setup (10 beetles released within wheat grains) were then poured in to a $400 \mathrm{~mL}$ plastic jar for a period of 30 days to see any weight loss in grains and the increases or decrease in the final population of these adults during this time. There were also four replications for this experiment to get mean values. Finally weight of wheat varieties was measured with sensitive electrical weighing balance to see any change in final weight due to insect feeding that was calculated in the form of percent weight loss.

Data recording: After a period of one month jars were examined to count the number of beetles in each replication by visual counting and separation of adults from wheat grains was done in glass petri dishes by using camel hair brush. Percent survival was calculated by using formula:

Survival $(\%)=\frac{\text { Number of live beetls }}{\text { Number of beetles released }} \times 100$

After separation of beetles, percent weight loss in final weight of grains was calculated by using formula:

Weight loss $(\%)=\frac{\text { Initial weight }- \text { Final weight }}{\text { Initial weight }} \times 100$

Data from number of grains, survival percentage and percent weight loss subjected to analysis of variance using Statistix software (version 8.1.). Means were compared by least significance difference test at $\mathrm{P}=0.05$.

\section{Results and Discussion}

Results showed that mean number of grains per $5 \mathrm{~g}$ weight was significantly different (Table $1 ; \mathrm{P}<0.05$ ) in five wheat varieties. Maximum mean number of 
grains was found in FARID (197.25 \pm 4.57$)$ followed by CHAKWAL $(179.00 \pm 4.23)$ being statistically similar. Minimum grains were in GALAXY (140.75 \pm 9.30) followed by MIRAJ (162.50 \pm 1.18$)$ and AASS $(166.00 \pm 10.00)$ in ascending order. Variety GALAXY had significantly less number of grains than that in FARID, CHAKWAL and AASS (Table 2, P $<0.05$; Fig. 1). Red flour beetles were unable to grow on whole wheat grains in any of five wheat varieties. Even their population was decreased from 10 adults released initially for 30 day period. Therefore, percent survival of beetles was calculated from 10 adults used for the experiment. Results showed that beetles' survival was non-significant in five varieties (Table 1; $\mathrm{P}>0.05$ ). Maximum survival was in CHAKWAL $(32.50 \pm 9.45 \%)$ followed by GALAXY $(15.00 \pm 4.08 \%)$, AASS $(7.50$ $\pm 1.58 \%)$, FARID $(7.50 \pm 3.02 \%)$ and MIRAJ $(0.00 \pm$ $0.00 \%$ ) in decreasing order (Table 2, Fig. 1). Finally we tested percent weight loss in grains of five varieties which showed that percent loss in grain weight was nominal and non-significant among varieties (Table 1; $P>0.05)$. Maximum weight loss occurred in AASS $(2.42 \pm 1.92 \%)$ followed by GALAXY $(2.42 \pm 0.31 \%)$, FARID $(2.17 \pm 0.77 \%)$, CHAKWAL $(0.92 \pm 0.53 \%)$ and MIRAJ $(0.33 \pm 0.21 \%)$ in decreasing order (Table 2, Fig. 1).

Significant difference in number of grains per unit weight ( $5 \mathrm{~g}$ in this study) can be due to difference in size of grains in different wheat varieties. It can be common that different wheat varieties have different size of grains. Thus a significant difference in number of grains per $5 \mathrm{~g}$ weight of five wheat varieties was obtained. These results are similar to other studies in which they found number of grains per unit weight varied significantly and a clear proportion of damaged and healthy grains per unit weight for different wheat varieties was established (Ali et al., 2009).

It is important to note that when the beetles, only sound grains were used as test medium and any damaged or broken grains were removed to check beetle's survival and weight loss in healthy grains of wheat varieties. In a 30 day period, beetles were unable to grow and reproduce on any of the five varieties of wheat. Results showed mortality of insects occurred in all varieties and survival out of initial ten insects in different varieties remained non-significant as counting of survived adults out of initial ten adults showed that their survival was non-significant in different wheat varieties. Maximum percent survival was $32.5 \%$ and minimum was $0.00 \%$. Red flour beetles are secondary pests of stored food

Table 1. Analysis of variance for number of grains, survival and weight loss by beetles for different wheat varieties

\begin{tabular}{|c|c|c|c|c|c|c|c|}
\hline \multirow[b]{2}{*}{ Source } & \multirow[b]{2}{*}{$\mathrm{DF}$} & \multicolumn{2}{|c|}{$\begin{array}{c}\text { Number of } \\
\text { grains }\end{array}$} & \multicolumn{2}{|c|}{$\begin{array}{c}\text { Survival } \\
(\%)\end{array}$} & \multicolumn{2}{|c|}{$\begin{array}{c}\text { Weight loss } \\
(\%)\end{array}$} \\
\hline & & $\mathrm{F}$ & $\mathrm{P}$ & $\mathrm{F}$ & $\mathrm{P}$ & $\mathrm{F}$ & $\mathrm{P}$ \\
\hline Variety & 4 & 7.68 & $0.0014 *$ & 2.61 & $0.0779 \mathrm{n} . \mathrm{s}$ & 0.79 & $0.5502 \mathrm{n}$ \\
\hline Error & 15 & & & & & & \\
\hline Total & 19 & & & & & & \\
\hline $\begin{array}{l}\text { Grand } \\
\text { mean }\end{array}$ & $\mathrm{CV}$ & 169.10 & 8.69 & 12.500 & 122.64 & 1.6480 & 131.96 \\
\hline
\end{tabular}

* = significance; $\mathrm{n} . \mathrm{s}=$ non-significant $\mathrm{DF}, \mathrm{F}$, and $\mathrm{P}=$ Survival $\%$ of no. of live beetles

Table 2. LSD all-pair wise comparisons tests of number of grains, survival (\%) and percent weight loss by red flour beetles in different wheat varieties

\begin{tabular}{llll}
\hline \hline Variety & Number of Grains & Survival (\%) & Weight loss (\%) \\
\hline FARID & $197.25 \pm 4.57 \mathrm{a}$ & $7.50 \pm 3.03 \mathrm{n} . \mathrm{s}$ & $2.42 \pm 0.32 \mathrm{n} . \mathrm{s}$ \\
CHAKWAL & $179.00 \pm 4.23 \mathrm{ab}$ & $32.50 \pm 9.45$ & $0.92 \pm 0.54$ \\
AASS & $166.00 \pm 10.00 \mathrm{~b}$ & $7.50 \pm 1.58$ & $2.42 \pm 1.92$ \\
MIRAJ & $162.50 \pm 1.18 \mathrm{bc}$ & $0.00 \pm 0.00$ & $0.33 \pm 0.21$ \\
GALAXY & $140.75 \pm 9.30 \mathrm{c}$ & $15.00 \pm 4.08$ & $2.42 \pm 0.32$ \\
\hline \hline
\end{tabular}

Alpha 0.05; n.s = non-significant; DF, F, and $\mathrm{P}=$ Survival $\%$ of no. of live beetles

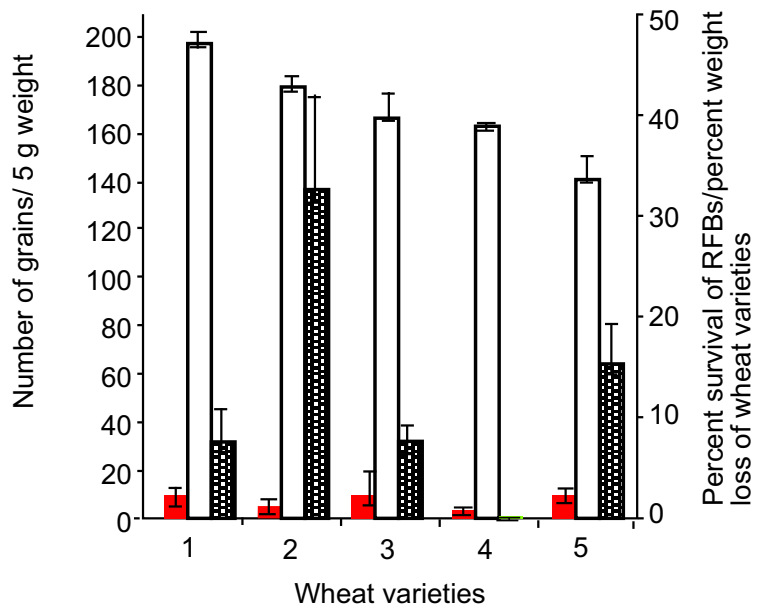

$\square$ No of grains $\square$ Percent loss 明 Survival

wheat varieties: 1-Farid, 2-Chakwal, 3-Aass, 4-Miraj, 5-Galaxy

Fig. 1. Number of grains, percent weight loss and percent survival of $T$. castaneum on different wheat varieties 
materials and are usually found infesting grain byproducts such as wheat flour and other farinaceous compounds. Their minute damage in sound grains may be detected and measured by using X-rays and other sensitive techniques (Karunakaran et al. 2004). It is not certain that their feeding on sound grains can leave prominent marks on those grains. Further finding damaged grains due to feeding by red flour beetles and other secondary stored grain insects having such weaker mouth parts may require special techniques as described by Karunakaren et al. (2004). Therefore alternatively percent weight loss in unit weight of wheat varieties over a given period of time can be an indication of their attack on sound grains. According to our results percent weight loss remained slight and non-significant on different wheat varieties and it ranged from 0.42 to 0.32 $\%$. These results show that red flour beetles being secondary stored grain insect pests caused minimal or negligible weight loss in sound wheat grains in five wheat varieties in a one month period and their pest status needs further evaluations for sound wheat grains keeping in view one month test period. Our results cannot be compared with Ali et al. (2009) firstly because they used different set of varieties and secondly the test period in their study was about five months in contrast with our studies which lasted for one month. A study was conducted by Renteria-Gutierez et al. (2000) to test the population growth of the lesser grain borer, Rhyzopertha dominica (F.) and the red flour beetle, T. castaneum (H.) under laboratory conditions. Both species were reared using whole grain and flour from four wheat varieties and four wheat groups. Although both species were able to complete development on both diet types e.g., whole grains and flour from four wheat varieties but $T$. castaneum adult populations was reduced on whole wheat grains compared with flour from four wheat varieties. Thus these results are in agreement with our current findings to some extent that T. castaneum population cannot sustain much on whole grains compared with flour. Further investigations would be beneficial in this research area for this cosmopolitan pest of wheat.

\section{Conclusion}

Current results show that red flour beetles could not induce major weight loss in thirty day period in wheat varieties containing little or no broken grains. Research should also be extended about investigation on red flour beetles population growth and damage in wheat containing different degree or percentage of cracked kernels to evaluate the effect of percentage of cracked kernels on damage or weight loss caused by these insects and on the population growth or survival percentage of red flour beetles and other secondary stored grain insect pests. Further it is also desirable to include more varieties under such investigation and testing period may be more then one month to see any weight loss to investigate resistance phenomenon in wheat against red flour beetles. It can help in eco-friendly management of such secondary stored grain insect pests and avoidance of residues due to pesticide usage in these commodities.

\section{References}

Ali, A., Sarwar, M., Khanzada, S., Abro, G.H. 2011. Evaluating resistance of wheat germplasms to attack by red flour beetle, Tribolium castaneum. $\mathrm{H}$ (Coleoptera). Pakistan Journal of Zoology, 43: 793-797.

Ali, A., Sarwar, M., Khanzada, S., Abro, G.H. 2009. Reaction of certain wheat varieties to the action of red flour beetle, Tribolium castaneum. H (Coleoptera) under insectary conditions. Pakistan Journal of Zoology, 41: 51-56.

Arbogast, R.T., Kendra, P.E., Mankin, R.W., McGovern, J.E. 2000. Monitoring insect pests in retail stores by trapping and spatial analysis. Journal of Economic Entomology, 93: 1531-1542.

Athanassiou, C. G., Opit, G. P., Throne, J. E. 2010. Influence of commodity type, percentage of cracked kernels, and wheat class on population growth of stored-product psocids (Psocoptera: Liposcelidae). Journal of Economic Entomology, 103: 985-990.

Bosly, H.A.Q., Kawanna, M.A. 2014. Fungi species and red flour beetle in stored wheat flour under Jazan region conditions. Toxicology and Industrial Health, 30: 304-310.

Gorham, J.R. 1979. The significance for human health of insects in foods. Annual Review of Entomology, 24: 209-224.

Gorham, J.R. 1975. Filth in foods: Implications for health. Journal of Milk and Food Technology, 38: 409-418.

Karunakaran, C., Jayas, D. S., White, N.D.G. 2004. Identification of wheat kernels damaged by red flour beetle using X-ray images. Biosystems Engineering, 87: 267-274.

Levinson, H. Z., Levinson, A. R., 1985. Storage and insect species of stored grain and tombs in ancient 
Egypt. Zeitschrift für angewandte Entomologie, 100: 321-339.

Phillips, T.W., Throne, J.E. 2010. Biorational approaches to managing stored product insects. Annual Review of Entomology, 55: 375-397.

Rees, D. 2007. Insects of Stored Grain. CSIRO Publishing, Canberra, Austration.

Renteria-Gutierez, T. R., Moreno-Martinez, E. J., BarronHoyos, M. 2000. Population growth of Rhyzopertha dominica (F.) and Tribolium castaneum (Herbst) in different wheat varieties and groups commercially produced in Sonora Mexico. Southwest Entomology, 25: 213-220.

Throne, J. E., Baker, J. E. Messina, F. J., Kramer, K. J.
Howard, J. A. 2000. Varietal Resistance, In: Alternatives to pesticides in stored-product IPM. Bh. Subramanyam and D. W. Hagstrum (eds.), pp. 166-192, Kluwer Academic Publishers, Boston, MA, USA.

Walter, V.E. 1990. Stored Product Pests. In: Handbook of Pest Control (K. and Story Moreland D. (eds.). OH, pp. 526-529. Franzak \& Foster Co., Cleveland, USA.

Weaver, D.K., Subramanyam, B. 2000. Botanicals. In: Alternatives to Pesticides in Stored Product, IPM, B.H. Subramanyam and D.W. Hagstrum (eds.). Kluwer Academic Publishers, Dordrecht, pp. 303320. 